\title{
PROBING COSMIC DUST OF THE EARLY UNIVERSE THROUGH HIGH-REDSHIFT GAMMA-RAY BURSTS
}

\author{
S. L. LiAng AND Aigen Li \\ Department of Physics and Astronomy, University of Missouri, Columbia, MO 65211, USA; shunlinliang@mizzou.edu, lia@missouri.edu \\ Received 2008 October 7; accepted 2008 November 11; published 2008 December 8
}

\begin{abstract}
We explore the extinction properties of the dust in the distant universe through the afterglows of high-redshifted gamma-ray bursts (GRBs) based on the "Drude" model which, unlike previous studies, does not require a prior assumption of template extinction laws. We select GRB 070802 at $z \approx 2.45$ (which shows clear evidence for the $2175 \AA$ extinction bump) and GRB 050904 at $z \approx 6.29$, the second most distant GRB observed to date. We fit their afterglow spectra to determine the extinction of their host galaxies. We find that (1) their extinction curves differ substantially from that of the Milky Way and the Small and Large Magellanic Clouds (which were widely adopted as template extinction laws in the literature); (2) the $2175 \AA$ extinction feature appears to be also present in GRB 050904 at $z \approx 6.29$; and (3) there does not appear to be strong evidence for the dependence of dust extinction on redshifts. The inferred extinction curves are closely reproduced in terms of a mixture of amorphous silicate and graphite, both of which are expected supernova condensates and have been identified in primitive meteorites as presolar grains originating from supernovae (which are considered as the main source of dust at high-z).
\end{abstract}

Key words: dust, extinction - galaxies: high-redshift - galaxies: ISM - gamma rays: bursts

\section{INTRODUCTION}

Dust is present in the high-redshift $(z>2)$ universe, as evidenced by the reddening of background quasars, the depletion of heavy elements in quasar absorption systems, and the farinfrared (IR) to millimeter thermal emission of distant quasars. Dust plays a crucial role in the formation and evolution history of stars and galaxies in the early universe. The importance of correcting for dust extinction in the universe is now widely recognized. In order to reveal the structure and evolution of the early universe, to use Type Ia supernovae $(\mathrm{SNe})$ as standard candles, and to infer the cosmological star-formation rate, it is essential to correct for the effects of dust extinction.

Gamma-ray bursts (GRBs), owing to their intense luminosity (emitting up to $\sim 10^{53} \mathrm{erg}$ ), allow their detection up to very high redshifts at $z \gtrsim 10$ (Lamb \& Reichart 2000). Particularly, the association of long-duration bursts with massive stars (and therefore with dusty regions of high-mass star formation) and the featureless, power-law-like spectral shapes of their afterglows, make GRBs an excellent probe of the dust at high redshifts.

In this Letter, we explore the dust extinction of the host galaxies of GRB 070802 at $z \approx 2.45$ and GRB 050904 at $z \approx 6.29$. We aim at a quantitative examination of the nature of the dust in the early universe and attempt to address one of the hotly debated questions in high- $z$ astrophysics: do the dust properties evolve as a function of redshift (particularly at $z>5$ where the dust source may be different)?

\section{DUST EXTINCTION MODEL}

We characterize the dust extinction properties of GRB hosts with the extinction quantity (e.g., $A_{V_{r}}$, the rest-frame visual extinction) and the wavelength dependence of the extinction (i.e., $A_{\lambda} / A_{V}$, or $A_{\nu} / A_{V}$ if expressed in frequency $v$, often known as the "extinction curve" or "extinction law"). We derive $A_{V_{r}}$ and $A_{\lambda} / A_{V}$ (or $A_{v} / A_{V}$ ) by fitting the ultraviolet (UV), optical, and near-IR afterglow photometry with a dust-reddened power-law model through

$$
F_{v}=F_{\mathrm{o}}(v / \mathrm{Hz})^{-\beta} \exp \left[-\frac{A_{V_{r}}}{1.086} \frac{A_{(1+z) v}}{A_{V_{r}}}\right],
$$

where $F_{v}$ is the afterglow photometry (with the Galactic foreground extinction corrected), $\beta$ is the intrinsic power-law slope of the afterglow, $F_{\mathrm{o}}$ is a normalization constant, $A_{(1+z) v}$ is the rest-frame extinction, and $z$ is the GRB redshift.

Unlike previous studies which often assume a template extinction law for $A_{\lambda} / A_{V}$ (i.e., the extinction curves of GRB hosts are assumed to resemble that of the Milky Way (MW), the Small Magellanic Cloud (SMC), the Large Magellanic Cloud (LMC), the "Calzetti" attenuation law of starburst galaxies (Calzetti et al. 1994), or the relatively flat "Maiolino" curve of active galactic nuclei (AGNs, Maiolino et al. 2001)), we take the "Drude" model proposed in Li et al. (2008a). This approach approximates the wavelength dependence of the extinction by a simple formula consisting of four dimensionless parameters $\left(c_{1}, c_{2}, c_{3}\right.$, and $\left.c_{4}\right)$ :

$$
\begin{aligned}
A_{\lambda} / A_{V}= & \frac{c_{1}}{(\lambda / 0.08)^{c_{2}}+(0.08 / \lambda)^{c_{2}}+c_{3}} \\
& +\frac{233\left[1-c_{1} /\left(6.88^{c_{2}}+0.145^{c_{2}}+c_{3}\right)-c_{4} / 4.60\right]}{(\lambda / 0.046)^{2}+(0.046 / \lambda)^{2}+90} \\
& +\frac{c_{4}}{(\lambda / 0.2175)^{2}+(0.2175 / \lambda)^{2}-1.95}
\end{aligned}
$$

where $\lambda$ is in $\mu \mathrm{m}$, the first term on the right-hand side represents the far-UV extinction rise, and the second term and the third term, respectively, account for the near-IR/visible extinction and the $2175 \AA$ extinction bump.

Compared to models based on template extinction curves, the "Drude" model is preferred because (1) it eliminates the need for a prior assumption of template laws-after all, there is no reason to assume that the "true" extinction curves of GRB hosts should resemble any of those templates, and (2) the analytical formula (Equation (2)) on which the "Drude" model is based restores the widely adopted MW, SMC, LMC, "Calzetti," and "Maiolino" templates-if the "true" extinction curve of a GRB 

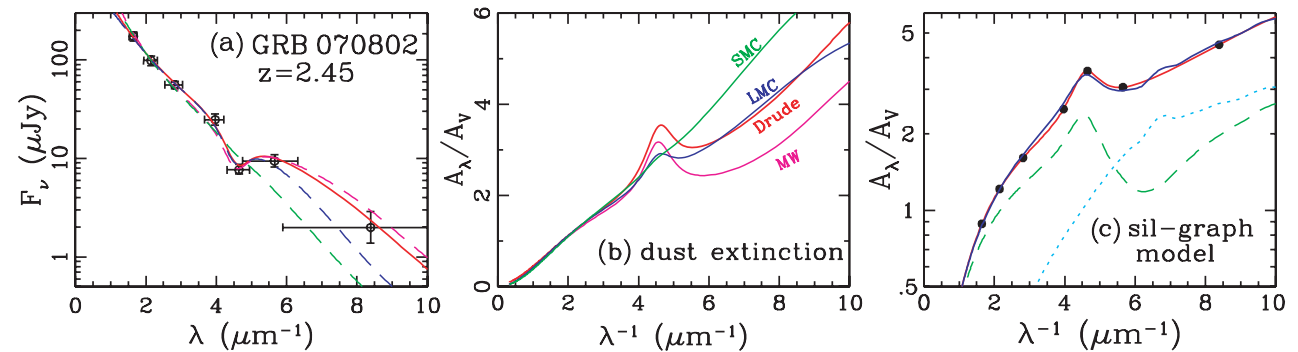

Figure 1. (a) Fitting the SED of the afterglow of GRB $070802(z \approx 2.45)$ with the "Drude" approach (red) and the MW (magenta), LMC (blue), and SMC (green) templates for the GRB host extinction curve. (b) Comparison of the MW (magenta), LMC (blue), and SMC (green) extinction laws with that derived from the "Drude" approach (red). (c) Fitting the derived extinction curve (red solid line and black filled circles) with a mixture of amorphous silicate (cyan dotted line) and graphite dust (green dashed line). The blue solid line plots the resulting model extinction curve.
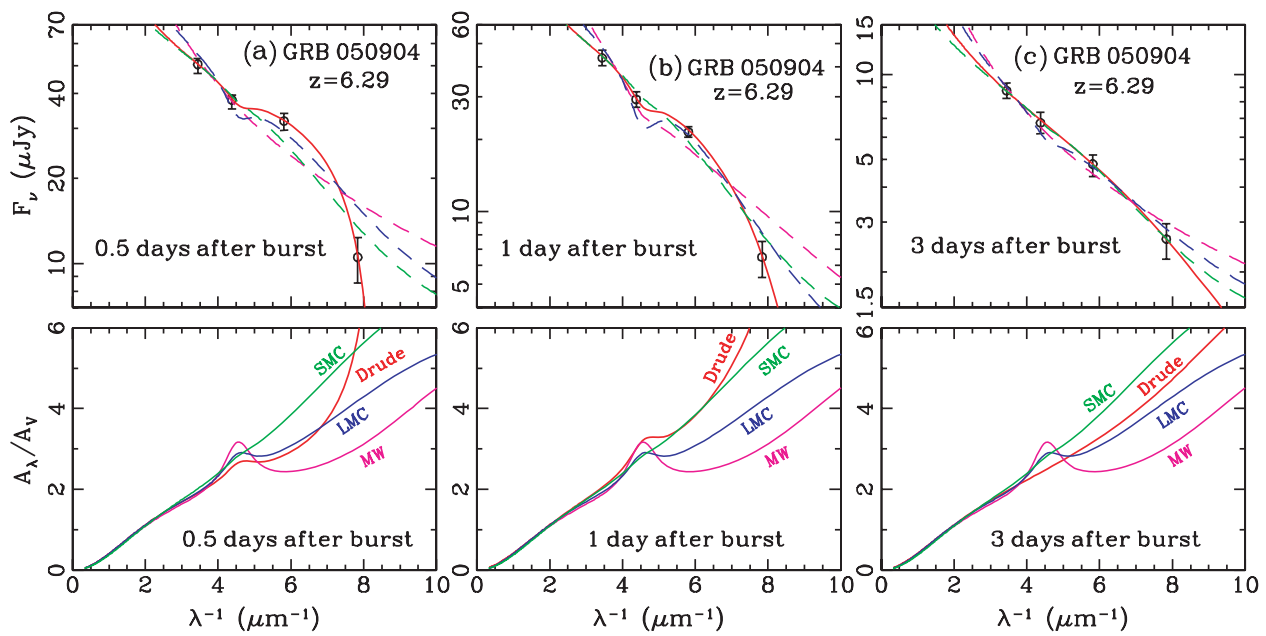

Figure 2. Same as Figures 1(a) and (b) but for GRB 050904 (Haislip et al. 2006; Tagliaferri et al. 2005) at three different epochs after burst.

host happens to resemble a certain template law, the "Drude" approach will allow us to restore it (see Li et al. 2008a).

\section{RESULTS}

We apply the "Drude" model to GRB 070802 at $z \approx 2.45$ and GRB 050904 at $z \approx 6.29$. They are selected for the following reasons: (i) they span a wide range of redshifts, from the moderately high redshift of $z \approx 2.45$ (GRB 070802) to the second highest redshift observed to date of $z \approx 6.29$ (GRB 050904); (ii) the afterglow photometry of GRB 070802 provides the most definite evidence for the presence of the $2175 \AA$ extinction feature in a GRB host galaxy (Krühler et al. 2008; Elíasdóttir et al. 2008); and (iii) the peculiar UKIRT $z$-band $\left(\lambda_{\text {rest }} \approx 1275 \AA\right.$ ) flux suppression of the GRB 050904 afterglow at 0.5 days and 1 day after the burst (Haislip et al. 2006; Stratta et al. 2007) was interpreted as evidence for an evolution of the dust properties at $z>6$ (Stratta et al. 2007).

Using Equations (1) and (2) and the Levenberg-Marquardt minimization algorithm, we fit the broadband spectral energy distributions (SEDs) of the afterglows of these $\mathrm{GRBs}^{1}$ with $\beta, A_{V}, c_{1}, c_{2}, c_{3}$, and $c_{4}$ allowed to vary as free parameters. ${ }^{2}$ Therefore, in the SED modeling we have six free parameters. ${ }^{3}$

\footnotetext{
1 For GRB 050904 we will consider three different epochs after the burst.

$2 F_{\mathrm{o}}$ is not really a free parameter; for a given set of $\left(\beta, A_{V}, c_{1}, c_{2}, c_{3}, c_{4}\right), F_{\mathrm{o}}$ is uniquely determined by the overall flux level.

3 Admittedly, the models based on template extinction laws have fewer parameters: with the shape of the extinction curve fixed, they only need to determine $\beta$ and $A_{V}$. The "Drude" approach needs four more parameters (i.e., $c_{1}, c_{2}, c_{3}$, and $c_{4}$ ) to describe the wavelength dependence of the extinction. This is the nature of the "Drude" approach; because of this the "Drude" approach is more flexible in revealing the "true" extinction curve.
}

It is unfortunate that the number of model parameters $\left(N_{\text {para }}=\right.$ 6) exceeds the number of photometry data points $N_{\text {data }}$ for GRB $050904\left(N_{\text {data }}=4\right.$ for all three epochs; Haislip et al. 2006; Tagliaferri et al. 2005). With $N_{\text {data }}=7$, GRB 070802 has a better wavelength coverage. ${ }^{4}$ We therefore use $\chi^{2} / N_{\text {data }}$ as a quality measure of the fit.

In Figure 1, we plot the "Drude" model fit to the afterglow SED of GRB 070802 as well as the derived extinction curve. The results for GRB 050904 at three different epochs after the burst are shown in Figure 2. We see in these figures that (1) the "Drude" model provides excellent fits to the observed SEDs; (2) the derived extinction curves differ substantially from the widely adopted template extinction laws; (3) the $2175 \AA$ extinction feature appears to be also present in the afterglow spectra of GRB 050904, the second most distant GRB observed to date, at epochs of 0.5 days and 1 day after the burst; (4) at an epoch of 3 days after the burst, the $2175 \AA$ feature appears to be absent in GRB 050904, suggesting that its carrier may have been destroyed by the burst ${ }^{5}$; and (5) there does not appear to be strong evidence for the dependence of dust extinction on redshifts (although the extinction curve does vary from one burst to another), as supported by a systematic study of $>20$ GRBs

\footnotetext{
4 For GRB 070802, we adopt the optical and near-IR photometry of Krühler et al. (2008) obtained by the 7-channel GRB Optical and Near-IR Detector (GROND) mounted on the $2.2 \mathrm{~m} \mathrm{ESO/MPI} \mathrm{Telescope.} \mathrm{The} \mathrm{ESO} \mathrm{VLT}$ spectroscopy of GRB 070802 is in close agreement with the GROND photometry (see Figure 5 of Elíasdóttir et al. 2008).

5 Indeed, one sees in Figure 2 a gradual flattening of the far-UV extinction rise from 0.5 days to 1 day and 3 days after burst, as expected from a preferential destruction of small grains responsible for the far-UV extinction by the burst (see Perna et al. 2003), that is reflected in Table 2 with a gradual increase (decrease) of the cutoff sizes (the power-law size distribution indices).
} 
Table 1

Parameters for Fitting the Afterglow SEDs with the "Drude" Model and the MW, LMC, and SMC Template Extinction Laws

\begin{tabular}{|c|c|c|c|c|c|c|c|c|}
\hline $\begin{array}{l}\text { Extinction } \\
\text { Type }\end{array}$ & $c_{1}$ & $c_{2}$ & $c_{3}$ & $c_{4}$ & $\begin{array}{c}A_{V} \\
(\mathrm{mag})\end{array}$ & $\beta$ & $\begin{array}{c}F_{\mathrm{o}} \\
(\mu \mathrm{Jy})\end{array}$ & $\chi^{2} / N_{\text {data }}$ \\
\hline \multicolumn{9}{|c|}{ GRB $070802(z \approx 2.54)$} \\
\hline Drude & 0.08 & 0.32 & -1.99 & 0.06 & 0.81 & 0.98 & 2.38E17 & 0.23 \\
\hline Drude & 0.10 & 0.34 & -1.98 & 0.00 & 0.83 & 0.97 & $1.70 \mathrm{E} 17$ & 1.86 \\
\hline MW & $\ldots$ & $\ldots$ & $\ldots$ & $\ldots$ & 0.81 & 1.39 & $9.39 \mathrm{E} 22$ & 0.84 \\
\hline SMC & $\ldots$ & $\ldots$ & $\ldots$ & $\ldots$ & 0.91 & 1.09 & $3.80 \mathrm{E} 18$ & 3.43 \\
\hline LMC & $\ldots$ & $\ldots$ & $\ldots$ & $\ldots$ & 1.57 & 0.002 & 628.3 & 0.66 \\
\hline \multicolumn{9}{|c|}{ GRB $050904(z \approx 6.29 ; 0.5$ days after burst $)$} \\
\hline Drude & 0.91 & 1.62 & -2.34 & 0.02 & 0.38 & 0.25 & 7.37E5 & 0.01 \\
\hline Drude & 0.86 & 1.73 & -2.30 & 0.00 & 0.42 & 0.27 & $1.43 \mathrm{E} 6$ & 1.20 \\
\hline MW & $\ldots$ & $\ldots$ & $\ldots$ & $\ldots$ & 0.01 & 1.42 & $1.12 \mathrm{E} 23$ & 5.51 \\
\hline SMC & $\cdots$ & $\ldots$ & $\cdots$ & $\cdots$ & 0.41 & 0.001 & 113.0 & 2.37 \\
\hline LMC & $\cdots$ & $\cdots$ & $\cdots$ & $\cdots$ & 0.46 & 0.35 & $2.23 \mathrm{E} 7$ & 3.94 \\
\hline \multicolumn{9}{|c|}{ GRB $050904(z \approx 6.29 ; 1$ day after burst) } \\
\hline Drude & 1.31 & 1.07 & -1.99 & 0.03 & 0.39 & 0.24 & $4.80 \mathrm{E} 5$ & 0.01 \\
\hline Drude & 1.54 & 1.13 & -1.99 & 0.00 & 0.46 & 0.26 & $9.41 \mathrm{E} 5$ & 1.12 \\
\hline MW & $\ldots$ & $\ldots$ & $\ldots$ & $\ldots$ & 0.16 & 1.73 & $6.11 \mathrm{E} 27$ & 5.58 \\
\hline SMC & $\cdots$ & $\ldots$ & $\ldots$ & $\cdots$ & 0.53 & 0.001 & 124.0 & 1.61 \\
\hline LMC & $\ldots$ & $\cdots$ & $\cdots$ & $\cdots$ & 0.84 & 0.03 & 601.0 & 2.07 \\
\hline \multicolumn{9}{|c|}{ GRB $050904(z \approx 6.29 ; 3$ days after burst $)$} \\
\hline Drude & 1.58 & 1.18 & -1.72 & 0.00 & 0.41 & 0.26 & 1.95E5 & 0.04 \\
\hline MW & $\ldots$ & $\ldots$ & $\ldots$ & $\ldots$ & 0.001 & 1.34 & $1.19 \mathrm{E} 21$ & 0.61 \\
\hline SMC & $\ldots$ & $\cdots$ & $\cdots$ & $\cdots$ & 0.33 & 0.16 & $4.10 \mathrm{E} 3$ & 0.06 \\
\hline LMC & $\cdots$ & $\cdots$ & $\cdots$ & $\cdots$ & 0.24 & 0.79 & $1.01 \mathrm{E} 13$ & 0.39 \\
\hline
\end{tabular}

at $z>2$ : the overall wavelength dependence of extinction, the steepness of the far-UV extinction rise, and the presence and strength of the $2175 \AA$ extinction bump do not appear to show any dependence on redshifts (S. L. Liang \& A. Li 2008, in preparation). The model parameters are tabulated in Table 1.

\section{DISCUSSION}

In deriving the extinction of GRB hosts, a major problem with the models based on template extinction laws is that the wavelength dependence of the extinction is fixed. For a featureless, power-law-like afterglow SED, this often leads to a preference of an SMC-type extinction and a small amount of $A_{V}$ (usually $<0.2 \mathrm{mag}$ ): obscured by an SMC-type extinction (which is roughly a power law: $A_{\lambda} \propto \lambda^{-1.2}$ ), an intrinsic powerlaw-like afterglow SED remains featureless and becomes a steeper power law. However, if the dust is "gray" (i.e., the extinction $A_{\lambda}$ only weakly varies with $\lambda$ ), the resulting dustobscured afterglow SED will still be a featureless power law, with the intrinsic power-law exponent unchanged. The possible presence of gray extinction has been suggested by a number of authors (e.g., see Savaglio et al. 2003; Savaglio \& Fall 2004; Stratta et al. 2004, 2005; Chen et al. 2006; Li et al. 2008b; Perley et al. 2008). The "Drude" approach allows us to break the degeneracy between "gray" extinction and SMCtype extinction.

Attempts have also been made to fit the afterglow SEDs with the MW, SMC, and LMC template extinction laws. As shown in Figures 1 and 2, no acceptable fits are obtained, except that the MW model for GRB 070802 and the SMC model for GRB 050904 at an epoch of 3 days after the burst fit the observed SEDs reasonably well. But even for these two cases, the "Drude" approach fits better as can be seen in Figures 1 and 2 and indicated by $\chi^{2} / N_{\text {data }}$ (see Table 1$)$.
Elíasdóttir et al. (2008) tried to fit the VLT/FORS2 spectroscopy and the GROND photometry with the Fitzpatrick \& Massa (1990; hereafter FM) parameterization as well as the MW-, LMC-, and SMC-type extinction. They found that satisfactory fits could be achieved only if one assumes a cooling break in the intrinsic spectrum, with the FM parameterization providing the best fit. However, one should caution that the FM parameterization is only valid for $\lambda<2700 \AA$, while the GROND photometry of GRB 070802 extends from $\sim 1400 \AA$ to $\sim 6400 \AA$ (in the GRB rest-frame).

The afterglow SEDs of the bursts discussed here all show a flux suppression at $\lambda \sim 4-6 \mu \mathrm{m}^{-1}$ and deviate appreciably from a power law (except for GRB 050904 at 3 days after the burst). As shown in Figures 1 and 2, the flux suppression is closely accounted for in terms of dust with a $2175 \AA$ bump in its extinction. For GRB 070802, the derived $2175 \AA$ bump is comparable to or even slightly stronger than that of the MW: for MW $c_{4} \approx 0.05$, while $c_{4} \approx 0.06$ for GRB 070802 . To validate the suggested detection of the $2175 \AA$ extinction feature, we have also tried to fit the afterglow SEDs with the "Drude" approach but setting $c_{4}=0$ (i.e., no $2175 \AA$ extinction bump). It is found that the fits (with $c_{4}=0$ ) are much worse, as reflected in the substantially increased $\chi^{2} / N_{\text {data }}$ (see Table 1).

The $2175 \AA$ bump, first detected by Stecher (1965), is the strongest spectroscopic interstellar extinction feature. This feature is seen in extinction curves along lines of sight in the MW and LMC. ${ }^{6}$ But it is rarely seen in the afterglow spectra of GRBs. So far, its possible detection is only reported in four bursts: GRB 970508 (Stratta et al. 2004), GRB 991216 (Kann et al. 2006; Vreeswijk et al. 2006), GRB 050802 (Schady et al. 2007), and GRB 070802 (Krühler et al. 2008), with the latter showing the clearest presence of the $2175 \AA$ extinction feature in its afterglow spectrum. In addition, Ellison et al. (2006) reported the detection of this feature in an intervening absorber at $z \approx 1.11$ toward GRB 060418. But the host galaxy of GRB 060418 at $z \approx 1.49$ seems to have an SMC-type extinction law.

The possible detection of the $2175 \AA$ extinction feature has been reported for a number of low, intermediate, and moderately high-redshift systems through (1) the composite absorption spectrum of intervening Mg II absorption systems (Malhotra 1997: $0.2<z<2.2$ ) or radio galaxies (Vernet et al. 2001: $z \sim 2.5)^{7}$; (2) the individual absorption spectra of intervening Mg II absorbers (Wang et al. 2004: $1.4<z<1.5$; Srianand et al. 2008: $z \sim 1.3)^{8}$; (3) the UV SEDs of massive, UVluminous star-forming galaxies (Noll \& Pierini 2005: $2<z<$ 2.5; Noll et al. 2007: $1<z<2.5$ ); and (4) the extinction curves of gravitational lensing galaxies (Toft et al. 2000: $z \approx 0.44$; Motta et al. 2002: $z \approx 0.83$; Wucknitz et al. 2003: $z \approx 0.93$; Muñoz et al. 2004: $z \approx 0.68$ ). However, Vijh et al. (2003) found that the dust in 906 Lyman break galaxies at $2<z<4$ does not

\footnotetext{
6 Most SMC extinction curves have no detectable $2175 \AA$ \&ump (Prévot et al 1984). But there exist regional variations in the SMC extinction curve. The SMC sight lines which show no $2175 \AA$ bump all pass through the SMC bar regions of active star formation (Prévot et al. 1984; Gordon \& Clayton 1998). The $2175 \AA$ bump is seen at least in one line of sight, Sk 143 (AvZ 456), which passes through the SMC wing, a region with much weaker star formation (Gordon \& Clayton 1998).

7 But York et al. (2006) found no evidence for the $2175 \AA$ bump in the composite absorption spectra of 809 intervening QSO Mg II absorbers at $1<z<1.9$.

8 The $2175 \AA$ extinction feature, the $9.7 \mu \mathrm{m}$ silicate absorption feature, and the diffuse interstellar bands are seen in the damped Ly $\alpha$ absorber at $z \approx 0.524$ toward the BL Lac object AO 0235+164 (Junkkarinen et al. 2004; Kulkarni et al. 2007).
} 

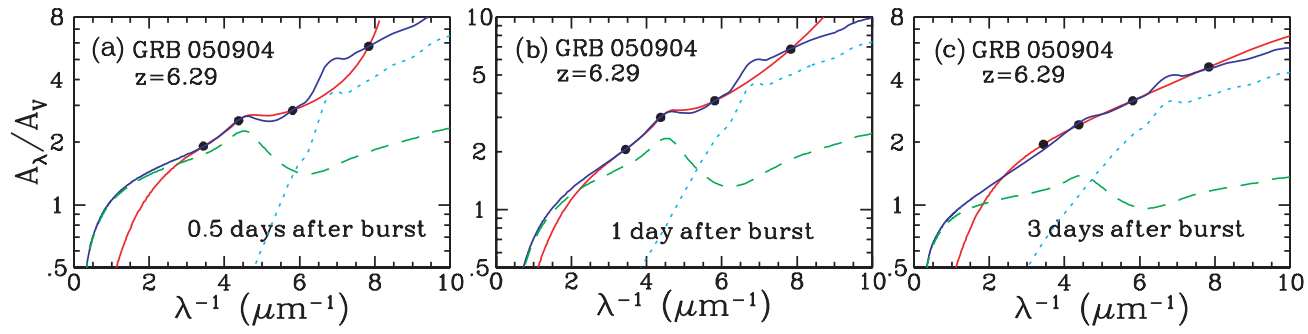

Figure 3. Same as Figure 1(c) but for GRB 050904 at three different epochs after burst.

exhibit the $2175 \AA$ extinction feature. This is probably related to the survival and destruction of the carriers of the $2175 \AA$ bump in different physical conditions.

Although the precise nature of the carrier of the $2175 \AA$ extinction feature remains unknown, it is generally accepted that it arises from small graphitic dust or a cosmic mixture of polycyclic aromatic hydrocarbon (PAH) molecules ( $\mathrm{Li} \&$ Draine 2001). In view of the detection of presolar graphite dust with a $\mathrm{SN}$ origin in primitive meteorites, it is not unreasonable to expect a $2175 \AA$ extinction bump for high- $z$ objects since the dust at $z>5$ is thought to originate from Type II SNe. On the other hand, PAHs have been detected in ultraluminous IR galaxies and submillimeter galaxies at $z>2$ through their vibrational bands at 6.2, 7.7, 8.6, and $11.3 \mu \mathrm{m}$ (see Lutz et al. 2005; Yan et al. 2005). PAHs were also seen in the Cloverleaf lensed QSO at $z \approx 2.56$ (Lutz et al. 2007). If PAHs are indeed responsible for the $2175 \AA$ extinction, it would not be surprising to see this feature in high- $z$ galaxies.

Finally, we fit the inferred extinction curves using a mixture of spherical amorphous silicate and graphite dust each with an exponential-cutoff power-law size distribution (e.g., see Kim et al. 1994):

$$
\begin{aligned}
A_{\lambda} / A_{V}= & A_{\text {sil }} \int_{a_{\min }}^{a_{\max }} C_{\text {ext }}^{\text {sil }}(a, \lambda) a^{-\alpha_{\text {sil }}} \exp \left(-a / a_{c, \text { sil }}\right) d a \\
& +A_{\text {gra }} \int_{a_{\text {min }}}^{a_{\text {max }}} C_{\text {ext }}^{\text {gra }}(a, \lambda) a^{-\alpha_{\text {gra }}} \exp \left(-a / a_{c, \text { gra }}\right) d a,
\end{aligned}
$$

where the lower (upper) cutoff size $a_{\min }\left(a_{\max }\right)$ is taken to be $50 \AA(1 \mu \mathrm{m})$ for both silicate and graphite dust; the powerlaw indices $\alpha_{\text {sil }}, \alpha_{\text {gra }}$ and the exponential-cutoff sizes $a_{\mathrm{c}, \text { sil }}$ and $a_{\mathrm{c}, \text { gra }}$ are treated as free parameters; $A_{\text {sil }}$ and $A_{\text {gra }}$ are related to the abundance of each species; and $C_{\mathrm{ext}}^{\text {sil }}\left(C_{\mathrm{ext}}^{\mathrm{gra}}\right)$ is the extinction cross-section of silicate (graphite) dust. As shown in Figures 1(c) and 3, the silicate-graphite model closely reproduces the inferred extinction curves for both GRBs, including the $2175 \AA$ extinction bump (see Table 2 for the size parameters). The major mismatch occurs at $\lambda \sim 7 \mu \mathrm{m}^{-1}$ which is probably due to the sudden rise of the silicate electronic absorption (see Kim \& Martin 1995). We note that both silicate and graphite are expected SN condensates (Todini \& Ferrara 2001; Nozawa et al. 2003). They have been identified as presolar grains in primitive meteorites originating from $\mathrm{SNe}$ which are considered as the main source of dust at $z>5$ (see Dwek et al. 2007).

By fitting the afterglow SEDs of GRB $050904(z \approx 6.29)$ with the extinction curve inferred for the distant BAL QSO at $z \approx 6.2$ (which displays a plateau at $\lambda^{-1} \sim 3.3-5.9 \mu \mathrm{m}^{-1}$, Maiolino et al. 2004), Stratta et al. (2007) argued that the dust properties may evolve beyond $z>5$. This seems to be supported by
Table 2

Dust Size Distributions for the Extinction Curves Derived from the "Drude" Model and a Mixture of Silicate and Graphite Grains

\begin{tabular}{lccccccc}
\hline \hline GRB & $z$ & $A_{\text {sil }}$ & $\alpha_{\text {sil }}$ & $a_{\text {c,sil }}(\mu \mathrm{m})$ & $A_{\text {gra }}$ & $\alpha_{\text {gra }}$ & $a_{\text {c, gra }}(\mu \mathrm{m})$ \\
\hline 070802 & 2.45 & 0.30 & 2.84 & 0.039 & 0.70 & 3.03 & 0.11 \\
050904 (0.5 days) & 6.29 & 0.59 & 3.08 & 0.014 & 0.41 & 3.10 & 0.33 \\
050904 (1 day) & 6.29 & 0.63 & 3.05 & 0.021 & 0.37 & 3.08 & 0.52 \\
050904 (3 days) & 6.29 & 0.68 & 3.00 & 0.045 & 0.32 & 2.88 & 0.76 \\
\hline
\end{tabular}

the dust at $z>5$ probably being produced by Type II SNe, while in the local universe asymptotic giant branch stars are a major source of dust. However, this study together with a preliminary analysis of $>20$ GRBs at $z>2$ based on the "Drude" approach does not indicate any dependence of the dust extinction on redshift. A more thorough and systematic study of the dust extinction and IR emission properties of high- $z$ GRBs is in progress, and will be used to further explore whether the dust properties vary as a function of redshift.

We thank V. P. Kulkarni, D. Malesani, S. Savaglio, G. Stratta, and D. Watson for very helpful comments and suggestions. We are supported in part by a NASA/Swift Theory Program, a NASA/Chandra Theory Program, and the NSFC Outstanding Overseas Young Scholarship.

\section{REFERENCES}

Calzetti, D., Kinney, A. L., \& Storchi-Bergmann, T. 1994, ApJ, 429, 582 Chen, S. L., Li, A., \& Wei, D. M. 2006, ApJ, 647, L13

Dwek, E., Galliano, F., \& Jones, A. P. 2007, Nuovo Cimento B, 122, 959

Elíasdóttir, Á., et al. 2008, ApJ, submitted (arXiv:0810.2897)

Ellison, S. L., et al. 2006, MNRAS, 372, L38

Fitzpatrick, E. L., \& Massa, D. 1990, ApJS, 72, 163

Gordon, K. D., \& Clayton, G. C. 1998, ApJ, 500, 816

Haislip, J. B., et al. 2006, Nature, 440, 181

Junkkarinen, V. T., Cohen, R. D., Beaver, E. A., Burbidge, E. M., Lyons, R. W., \& Madejski, G. 2004, ApJ, 614, 658

Kann, D. A., Klose, S., \& Zeh, A. 2006, ApJ, 641, 993

Kim, S.-H., \& Martin, P. G. 1995, ApJ, 442, 172

Kim, S.-H., Martin, P. G., \& Hendry, P. D. 1994, ApJ, 422, 164

Krühler, T., et al. 2008, ApJ, 685, 376

Kulkarni, V. P., York, D. G., Vladilo, G., \& Welty, D. E. 2007, ApJ, 663, L81

Lamb, D. Q., \& Reichart, D. E. 2000, ApJ, 536, 1

Li, A., \& Draine, B. T. 2001, ApJ, 554, 778

Li, A., Liang, S. L., Kann, D. A., Wei, D. M., Klose, S., \& Wang, Y. J. 2008a, ApJ, 685, 1046

Li, Y., Li, A., \& Wei, D. M. 2008b, ApJ, 678, 1136

Lutz, D., Valiante, E., Sturm, E., Genzel, R., Tacconi, L. J., Lehnert, M. D., Sternberg, A., \& Baker, A. J. 2005, ApJ, 625, L83

Lutz, D., et al. 2007, ApJ, 661, L25

Maiolino, R., et al. 2001, A\&A, 365, 28

Maiolino, R., et al. 2004, Nature, 431, 533

Malhotra, S. 1997, ApJ, 488, L101 
Motta, V., et al. 2002, ApJ, 574, 719

Muñoz, J. A., Falco, E. E., Kochanek, C. S., McLeod, B. A., \& Mediavilla, E. 2004, ApJ, 605, 614

Noll, S., \& Pierini, D. 2005, A\&A, 444, 137

Noll, S., Pierini, D., Pannella, M., \& Savaglio, S. 2007, A\&A, 472, 455

Nozawa, T., Kozasa, T., Umeda, H., Maeda, K., \& Nomoto, K. 2003, ApJ, 598, 785

Perley, D. A., et al. 2008, ApJ, 672, 449

Perna, R., Lazzati, D., \& Fiore, F. 2003, ApJ, 585, 775

Prévot, M. L., et al. 1984, A\&A, 132, 389

Savaglio, S., \& Fall, S. M. 2004, ApJ, 614, 293

Savaglio, S., Fall, S. M., \& Fiore, F. 2003, ApJ, 585, 638

Schady, P., et al. 2007, MNRAS, 377, 273

Srianand, R., Gupta, N., Petitjean, P., Noterdaeme, P., \& Saikia, D. J. 2008, MNRAS, 391, L69

Stecher, T. P. 1965, ApJ, 142, 1683
Stratta, G., Fiore, F., Antonelli, L. A., Piro, L., \& De Pasquale, M. 2004, ApJ, 608,846

Stratta, G., Maiolino, R., Fiore, F., \& D’Elia, V. 2007, ApJ, 661, L9

Stratta, G., Perna, R., Lazzati, D., Fiore, F., Antonelli, L. A., \& Conciatore, M. L. 2005, A\&A, 441, 83

Tagliaferri, G., et al. 2005, A\&A, 443, L1

Todini, P., \& Ferrara, A. 2001, MNRAS, 325, 726

Toft, S., Hjorth, J., \& Burud, I. 2000, A\&A, 357, 115

Vernet, J., Fosbury, R. A. E., Villar-Martin, M., Cohen, M. H., Cimatti, A., di Serego Alighieri, S., \& Goodrich, R. W. 2001, A\&A, 366, 7

Vijh, U. P., Witt, A. F., \& Gordon, K. D. 2003, ApJ, 587, 533

Vreeswijk, P. M., et al. 2006, A\&A, 447, 145

Wang, J., Hall, P. B., Ge, J., Li, A., \& Schneider, D. P. 2004, ApJ, 609, 589

Wucknitz, O., Wisotzki, L., Lopez, S., \& Gregg, M. D. 2003, A\&A, 405, 445

Yan, L., et al. 2005, ApJ, 628, 604

York, D. G., et al. 2006, MNRAS, 367, 945 